\title{
Phonocardiographic Study of Hyperthyroidism
}

\author{
Hideo UEDA, M.D., Zen'ichiro Uozumi, M.D., \\ Hiroshi Watanabe, M.D., Tohru Kobayashi, M.D., \\ Nobuyoshi Kawai, M.D., Tohru Matuura, M.D., \\ and Tohru IWAsE, M.D.
}

The variability of the phonocardiographic feature in hyperthyroidism was described. The first heart sound was accentuated, but the Q-I interval was within the normal range. The mechanical systolic period was reduced out of proportion to the heart rate. The second heart sound frequently splitted, and its pulmonic component was accentuated. The third heart sound was accentuated, but the fourth heart sound was inaudible except in 3 cases with presystolic click. The systolic murmur was found in all cases, and it was an early peaked ejection systolic murmur irrespective of area of maximal intensity. A mid-diastolic murmur was present at the apex in $17 \%$ of cases revealing enlarged heart in the chest roentgenogram and myocardial damage pattern in the electrocardiogram. One case had two varieties of diastolic murmur, left-and right-sided Carey Coombs murmur.

TN the case of hyperthyroidism, the snappy loud first heart sound at the apex and the functional systolic murmur at the pulmonic area are well-known auscultatory findings. Furthermore, the apical diastolic murmur ${ }^{1-3)}$ or diastolic gallop ${ }^{2-4)}$ and loud pulmonic second heart sound ${ }^{5)}$ are audible in some cases. These auscultatory findings may simulate those of mitral valve disease, especially when atrial fibrillation and congestive heart failure are present in combination with hyperthyroidism.

The purpose of this report is to analyse the details of the heart sounds and murmurs in hyperthyroidism in relation to clinical findings, and to compare them with those in normal subjects and cases of mitral valve disease.

\section{Materials And Methods}

Fourty-two cases with hyperthyroidism aged 15 to 58 were studied. In 9 cases follow-up study was made after effective treatment. Thus a total of 52

From the Second Department of Internal Medicine, Faculty of Medicine, University of Tokyo, Tokyo. 
phonocardiograms were analysed.

Using a multi-filter system phonocardiograph and a dynamic microphone, ${ }^{6}$ the recordings were made in supine position at the end of ordinary expiration. Usually the Medium Phonocardiogram (PCG), and, if necessary, other PCG was utilized for measurement to compare with the normal data, which had already been reported. ${ }^{73,8)}$ The details of analysis will be mentioned in respective item.

The phonocardiographic findings were correlated to basal metabolic rate and/or $I^{131}$ uptake, which were used as an index of clinical severity of hyperthyroidism. The chest roentgenogram and the electrocardiogram (ECG) were also referred to some of the phonocardiographic findings.

\section{Results And Comments}

1. Q-I interval :

The interval between the onset of the $Q$ wave in ECG and the onset of the major deflection of the first heart sound (IS) was within normal range $(0.03$ to $0.06 \mathrm{sec}$.) in all cases including 6 cases with atrial fibrillation. Fig. 1 shows the distribution of the Q-I interval in hyperthyroidism as compared with that in mitral stenosis, in which the delayed appearance of IS is one of characteristic features.

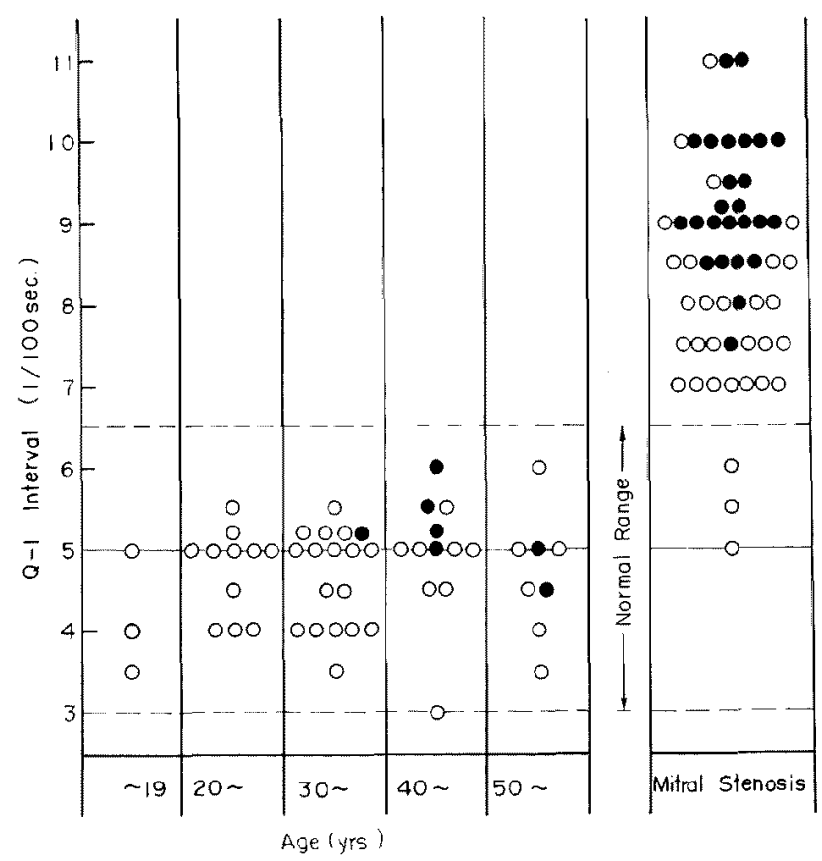

Fig. 1. Distribution of Q-I interval of hyperthyroidism, as compared with that of mitral stenosis. Black circle indicates the cases with atrial fibrillation. 
2. Loudness of IS :

Apical IS was accentuated in relative loudness to the second heart sound (II S) as well as in absolute loudness estimated by the calibration method.

Even at the apex, II S was louder than IS in about two-thirds of normal subjects, as previously reported. In contrast, I S was louder than II $S$ in all but 3 cases of hyperthyroidism. Moreover, the amplitude of I S was over three times greater than that of II S in about one-third of total cases.

The intensity ratio of IS, indicated by the ratio of the amplitude of IS to the height of calibration wave, was also increased. In relationship between the intensity ratio of IS and the basal metabolic rate, there was no definite correlation, but it may be safe to say that highly increased intensity ratio of IS was found in cases with highly elevated basal metabolic rate (Fig. 2).

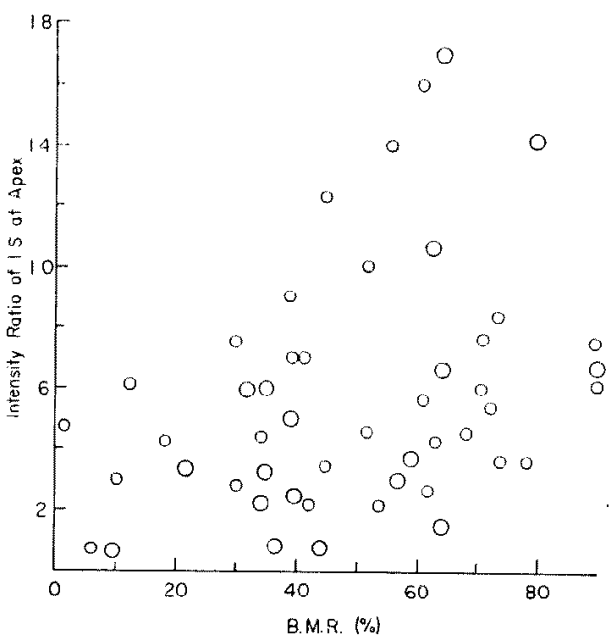

Fig. 2. Relation of intensity ratio of apical IS to basal metabolic rate. Small circle indicates the cases below the age of 39 and large circle indicates the cases above the age of 40 . (This division according to the size of circle is used in following figures.)

In comparison of intensity ratio of IS in Medium PCG with that in High PCG, the latter was greater than the former in most cases. This fact suggests that the accentuation of IS is due to an increase not only in intensity but in pitch.

The accentuation of IS in hyperthyroidism might result from tachycardia, but there was no definite correlation between the intensity ratio of IS and the heart rate. A reasonable factor of this accentuation may 
be the rapid closure of mitral valve caused by increased contractility of the heart muscle which may be elicited by the direct action of thyroid hormone.

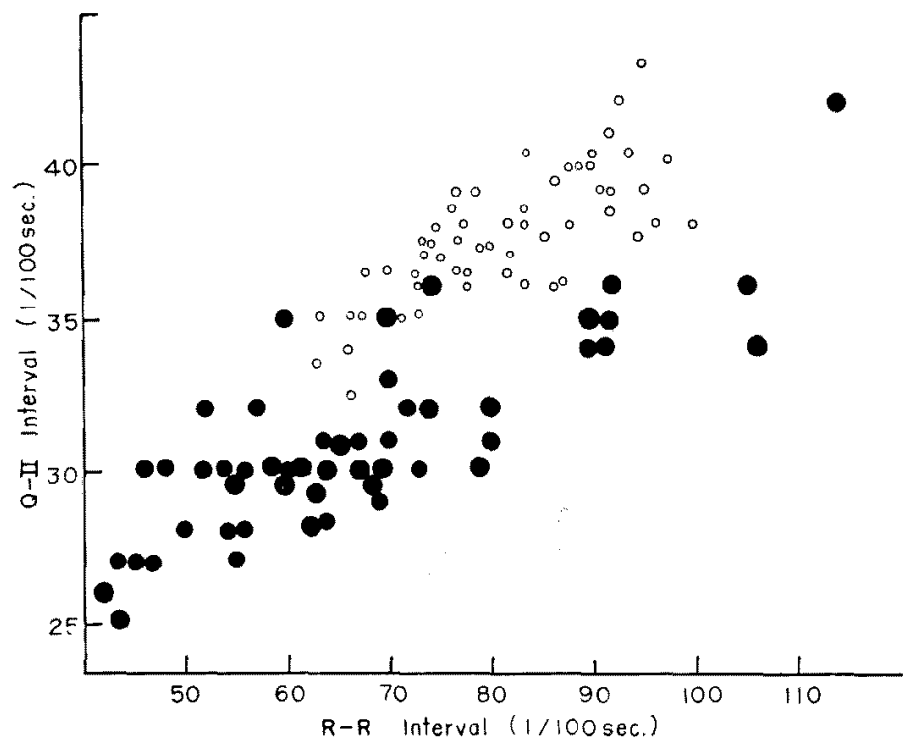

Fig. 3a. Relation of $Q-I I$ interval to $R-R$ interval in hyperthyroidism (expressed by black circle), as compared with that in normal subjects (expressed by the smallest open circle).

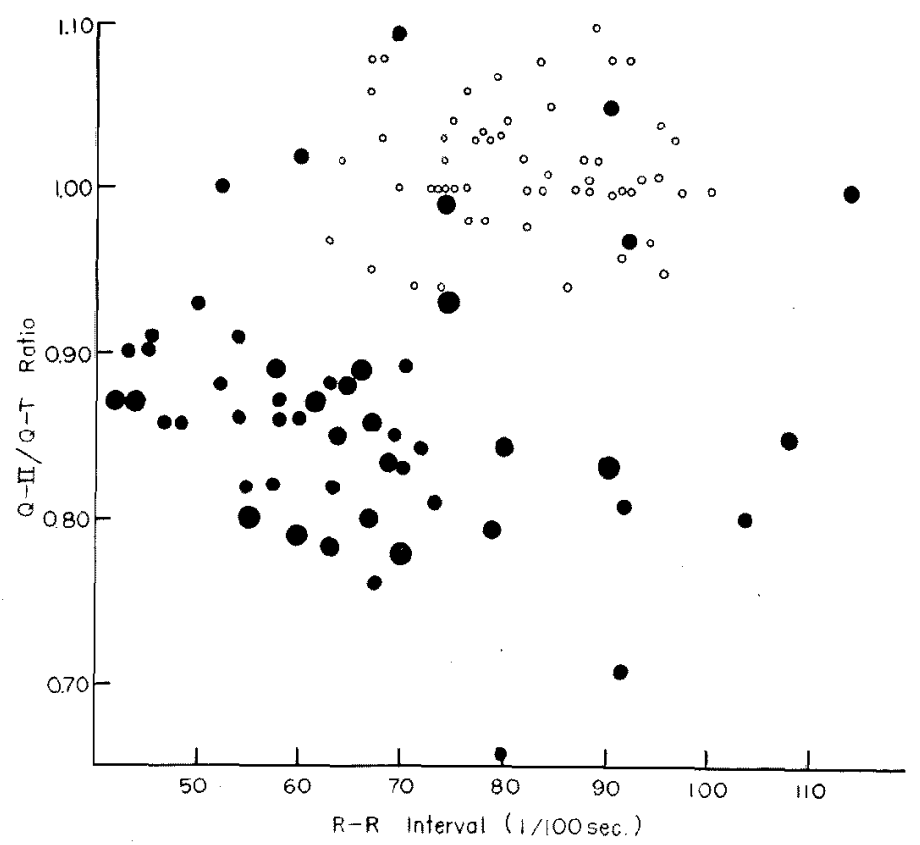

Fig. 3b. Relation of $Q-I I / Q T$ ratio to $R-R$ interval. The same symbol as in Fig. 3a is used. 
3. Q-II interval :

The duration of ventricular systole, indicated by the interval between the onset of the $Q$ wave in ECG and the onset of the major deflection of IIS, was shortened out of proportion to the heart rate, as seen in Fig. 3 a.

The reduction of the mechanical systole (Q-II interval) was more marked than that of the electrical systole (Q-T interval), because the latter of our cases tended to prolong within or out of normal range. Fig. $3 \mathrm{~b}$ shows the relationship between the systolic quotient (the ratio of the Q-II interval to the Q-T interval) and the R-R interval.

The so-called Hegglin's syndrome, ${ }^{9}$ in which the Q-II interval was over $0.04 \mathrm{sec}$. shorter than the Q-T interval, was observed in $58 \%$ of total cases, including 7 cases with abnormally prolonged Q-T interval.

There was no significant correlation between the systolic quotient and the severity of hyperthyroidism, but it may be worthwhile to mention that the shortening of the mechanical systole was marked in most cases of severe hyperthyroidism.

4. Splitting and loudness of II S :

The splitting of II S, the interval of which ranged from 0.02 to 0.05 sec. during expiration, was found in $69 \%$ of total cases.

The incidence of the splitting of II S in normal subjects decreased rapidly after the age of 40 years, whereas it was found in $63 \%$ of cases of hyperthyroidism among the same age group.

The intensity ratio of the aortic component of splitted IIS at the base was within normal range. On the other hand, the pulmonic component of II S was louder than or equal to the aortic one in most cases with the splitting of II S, as shown in Fig. 4. Accordingly the accentuation of II $\mathrm{S}$ on auscultation in hyperthyroidism was due to the accentuation of the pulmonic component of II S.

The high incidence of the splitting of II S may be explained by the discrepancy of the mechanical systole between the left and right ventricles, which may result from a decrease of systemic peripheral resistance and a slight increase or no change of pulmonic vascular resistance. ${ }^{10)}$ The accentuation of the pulmonic component of II S may be due, in a part, to the increased pulmonic vascular resistance and due, in another part, to the dilatation of pulmonary artery.

5. Third heart sound and diastolic murmur:

The third heart sound (IIIS) was accentuated and it was clearly recorded in Medium PGG or up to higher frequency PCG in $67 \%$ of total cases. In other words, two-thirds of cases showed the grade 3 or more of accentuation ${ }^{6)}$ of III S. Fig. 6 shows the incidence of respective grade of accentuation of III $S$ in hyperthyroidism, as compared with that 

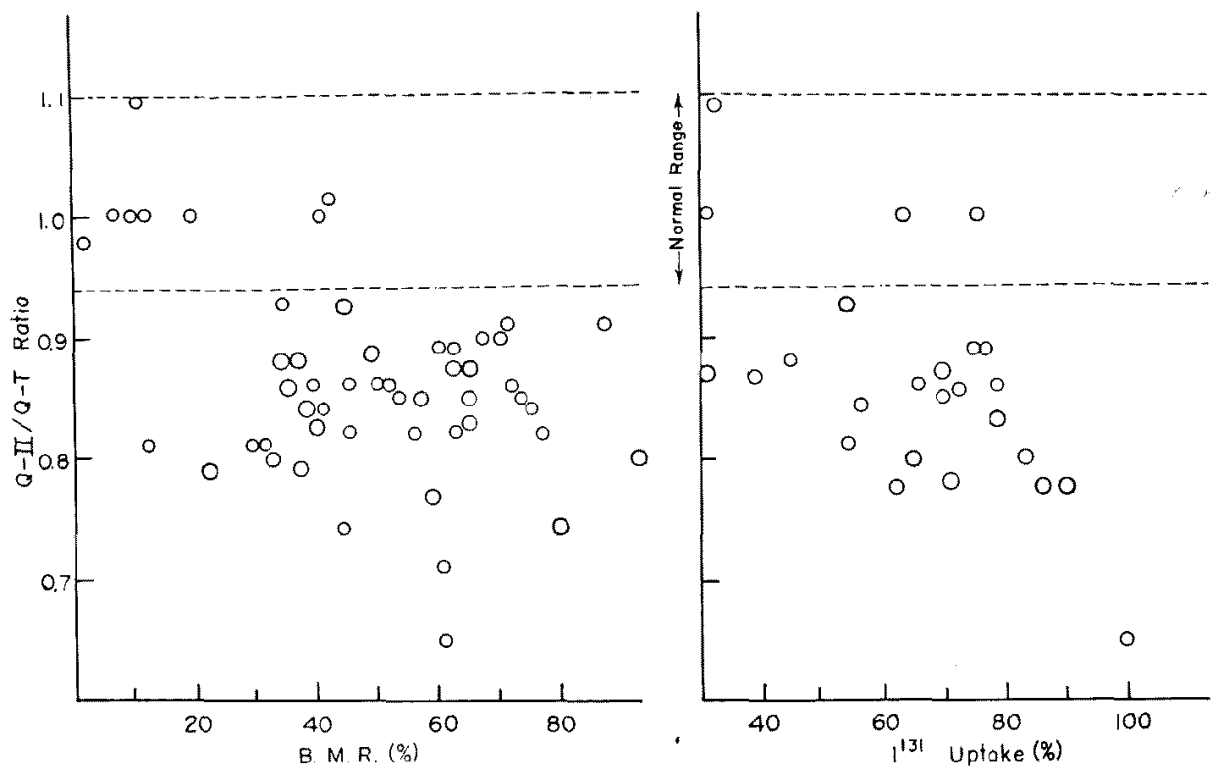

Fig. 4. Relation of Q-II/Q-T ratio to basal metabolic rate and $I^{131}$ uptake.

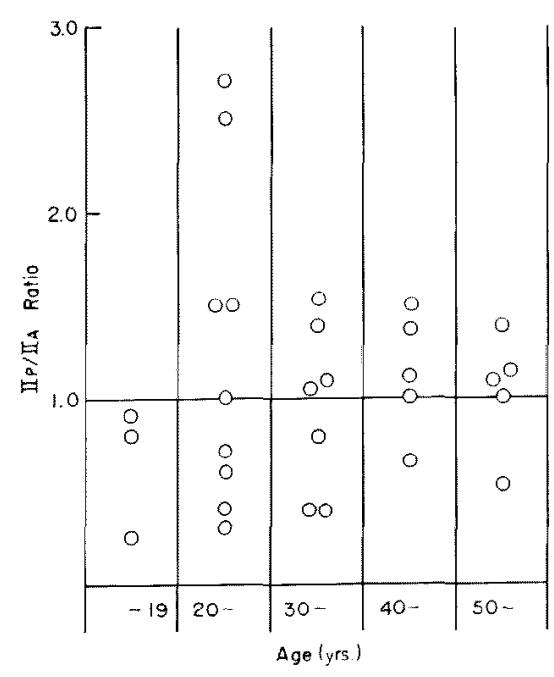

Fig. 5. Distribution of amplitude ratio of the pulmonic component to the aortic component of splitted II S at the base.

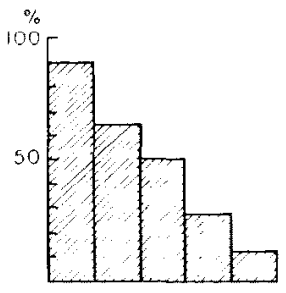

$<39$ yrs.
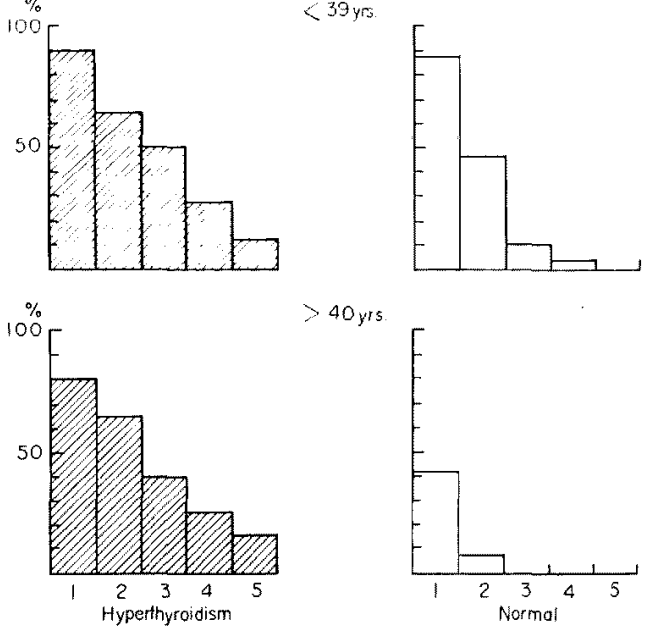

Fig. 6. Incidence of cases with respective grade of accentuation of III $\mathrm{S}$ in hyperthyroidism, as compared with that in normal subjects.

in normal subjects. As previously reported, ${ }^{11)}$ young normal subjects had not infrequently loud III S, which was difficult to distinguish graphically from IIIS of pathological condition. On the other hand, in the cases 
above the age of 40 , III $\mathrm{S}$ was pathological when it was audible and/or when it was recorded up to the Medium PCG. Therefore, the accentuation of III S should be discussed under consideration of the age.

The relationship between the accentuation of III $S$ and basal metabolic rate indicated that highly accentuated III $\mathrm{S}$ tended to be found in severe cases (Fig. 7).

The accentuation of III S had also a trend to be found in the cases

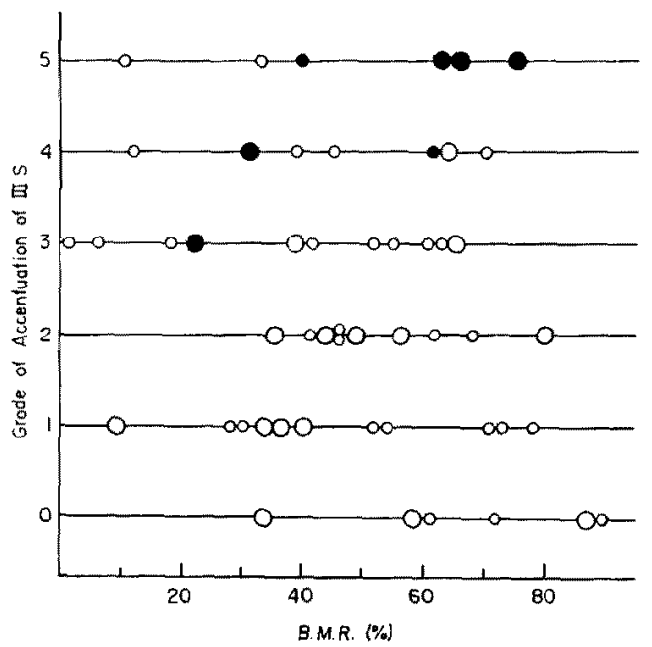

Fig. 7. Relation of grade of accentuation of III $S$ to basal metabolic rate. Black circle indicates the cases with the mid-diastolic murmur. (The same symbol is used in Figs. 8 and 9.)

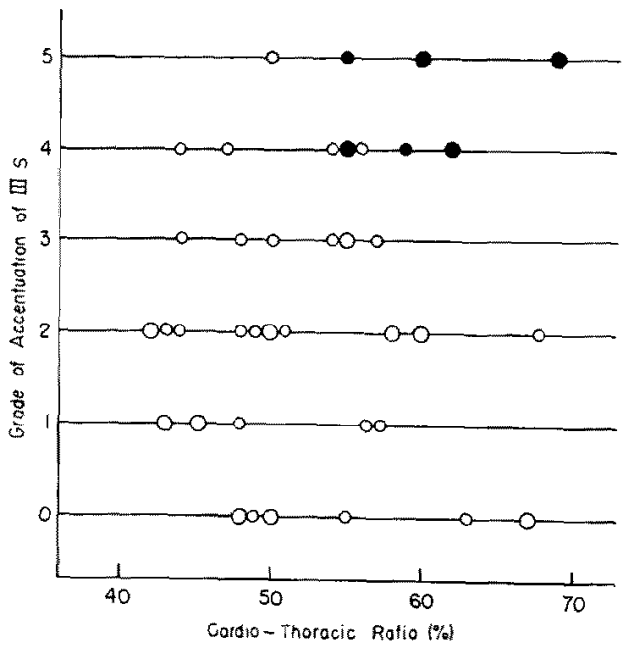

Fig. 8. Relation of grade of accentuation of III $S$ to cardio-thoracic ratio. 


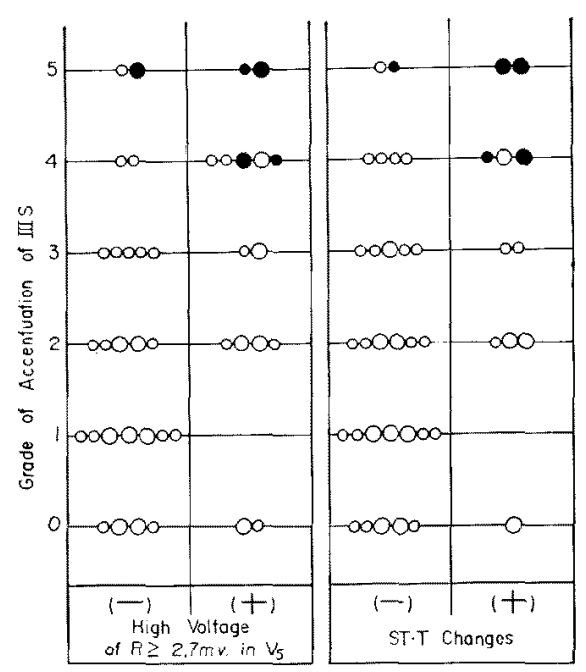

E CG Findings

Fig. 9. Relation of grade of accentuation of III $S$ to ECG findings.

with enlarged heart and ECG changes such as high voltage of $\mathrm{R}$ wave in lead $V_{5}$ and ST-T depression, as seen in Figs. 8 and 9.

In regard to the diastolic murmur in hyperthyroidism, some authors ${ }^{5}$, 12) stressed that it was hardly observed in any cases, and the others ${ }^{13), 14)}$ described that the murmur was of presystolic timing. However, in our cases, the mid-diastolic murmur was heard in low intensity of Levine 1 or 2, and it was recorded in 7 of all cases (17\% of total cases), which showed enlargement of the heart and ECG changes. A cause of production of this murmur had been explained by an increase of blood flow and rapid flow of the blood through the mitral valve orifice. ${ }^{1)}$ However, in addition to this absolutely increased flow load, there was another important factor, that was relatively increased overload on the damaged myocardium as suggested clinically from the presence of enlarged heart and ECG changes.

The graphic appearance of the mid-diastolic murmur fell in a category of the murmur of relative mitral stenosis or the Carey Coombs murmur. ${ }^{5)}$ But the murmur tended to be higher-pitched and of longer duration than that observed in acute rheumatic carditis, though it started from or immediately after III S.

6. Fourth heart sound:

The fourth heart sound (IV S) was inaudible except in 3 cases, which had presystolic $\operatorname{click}^{15)}$ at the third or fourth intercostal space on the left sternal border. However, IV S in hyperthyroidism was somewhat accentuated as compared with that in normal subjects, as seen in Fig. 10. 

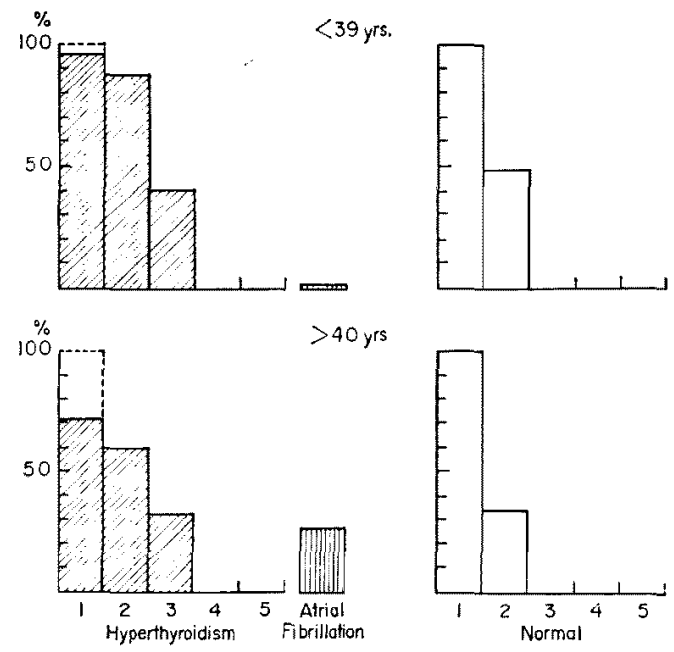

Fig. 10. Incidence of cases with respective grade of accentuation of IV S in hyperthyroidism, as compared with that in normal subjects.

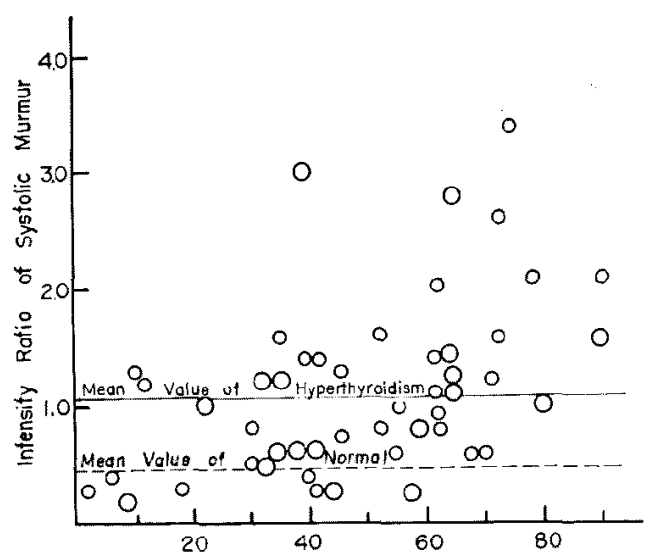

B. M. R.

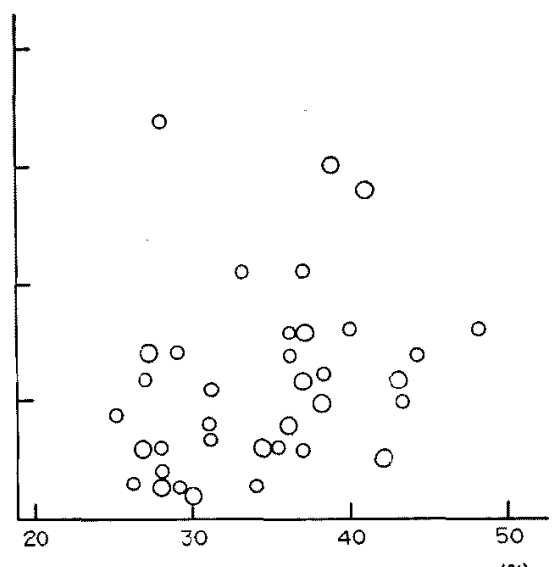

Enlargement Grade of Pulmonary Conus $(\%)$

Fig. 11. Relation of intensity ratio of systolic murmur to basal metabolic rate and grade of enlargement of pulmonary conus.

\section{Systolic murmur :}

The systolic murmur was heard in a range of grade 2 to 4 , and it was rough or harsh, and sometimes scratchy in quality. The area of maximal intensity was usually at the pulmonic or Erb's area, but in about a quarter of total cases, especially among the elder subjects, it was observed in the area downward to the apex of the heart. The graphic appearance of the murmur was the ejection systolic murmur ${ }^{16)}$ irrespective of its area of maximal intensity, which began in early systole showing a crescendo-decrescendo configuration and ended before II S. 


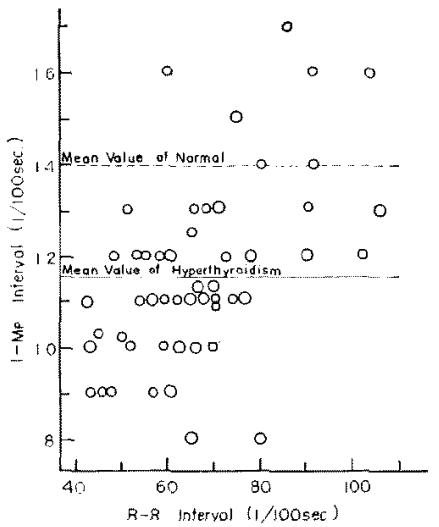

Fig. 12. Relation of interval between $I S$ and intensity peak of systolic murmur to $R \cdot R$ interval.

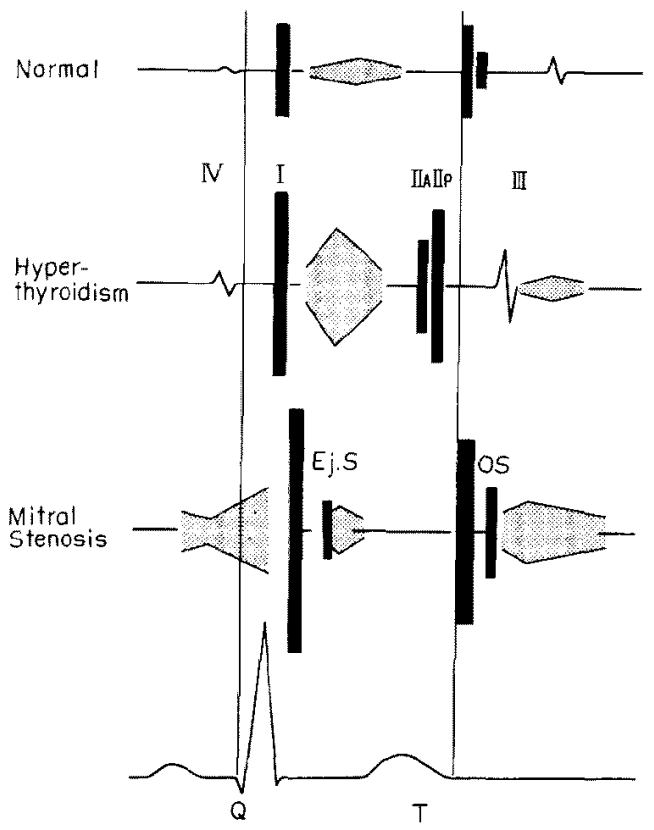

Fig. 13. Schematic representation of PCG findings in hyperthyroidism, as compared with those in normal and mitral stenosis. PCG findings in hyperthyroidism are as follows: Accentuation of I S, normal Q-I interval, reduced Q-II interval, splitting of II $\mathrm{S}$, accentuation of the pulmonic component of II $\mathrm{S}$, early peaked ejection systolic murmur and accentuated III $S$ followed by mid-diastolic murmur.

The intensity ratio of the murmur, calculated by an amplitude ratio of peak intensity of the murmur to the calibration wave, was increased and greatly increased ratio was observed in severe cases. There was some degree of correlation between the intensity ratio of the murmur and the 
grade of enlargement of pulmonary conus, which was estimated by the chest roentgenogram using the ratio of the distance from the middle line of thoracic cage to the second arch of left cardiac border to the semidiameter of thoracic cage.

Fig. 11 shows the relations of the intensity ratio of the systolic murmur to the basal metabolic rate and to the grade of enlargement of pulmonary conus.

The intensity peak of the murmur usually situated in early systole. However, the timing of the intensity peak was affected mainly by heart rate as shown in Fig. 12. The ratio of the interval between IS and intensity peak to the interval between IS and II S fell nearly within the normal range.

8. Miscellaneous findings :

Thyroid bruit was found in about a half of total cases. The cases with thyroid bruit was mostly severe cases, but the reverse was not true.

We have had no experience of the case with regurgitant diastolic murmur of the semilunar valves due to hyperthyroidism. ${ }^{17)}$

Fig. 13 shows the schematic representation of above-mentioned phonocardiographic findings in hyperthyroidism, as compared with those in normal subjects and in cases of mitral stenosis.

\section{Illustrative Gases}

Case 1. A 26-year-old man suffered from palpitation, weight loss and hand

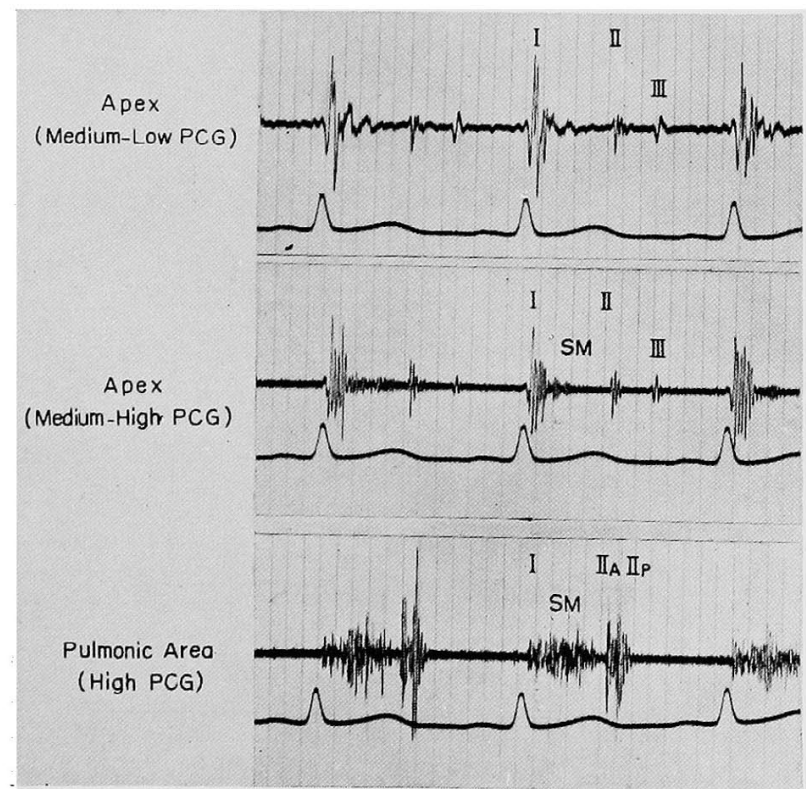

Fig. 14. PCG of a 26-year-old man, showing the typical findings. Basal metabolic rate was $+79 \%$. 
tremor for 3 years. Laboratory data disclosed basal metabolic rate $+79 \%$, I 131 uptake $73 \%$, and protein bound iodine $11.7 \gamma / 100 \mathrm{ml}$. PGG of Fig. 14 shows the followings: At the apex, I S was accentuated and III S was clearly recorded up to the High PCG (grade of accentuation, 5). At the pulmonic area, there were an early peaked ejection systolic murmur and the splitted II S, of which

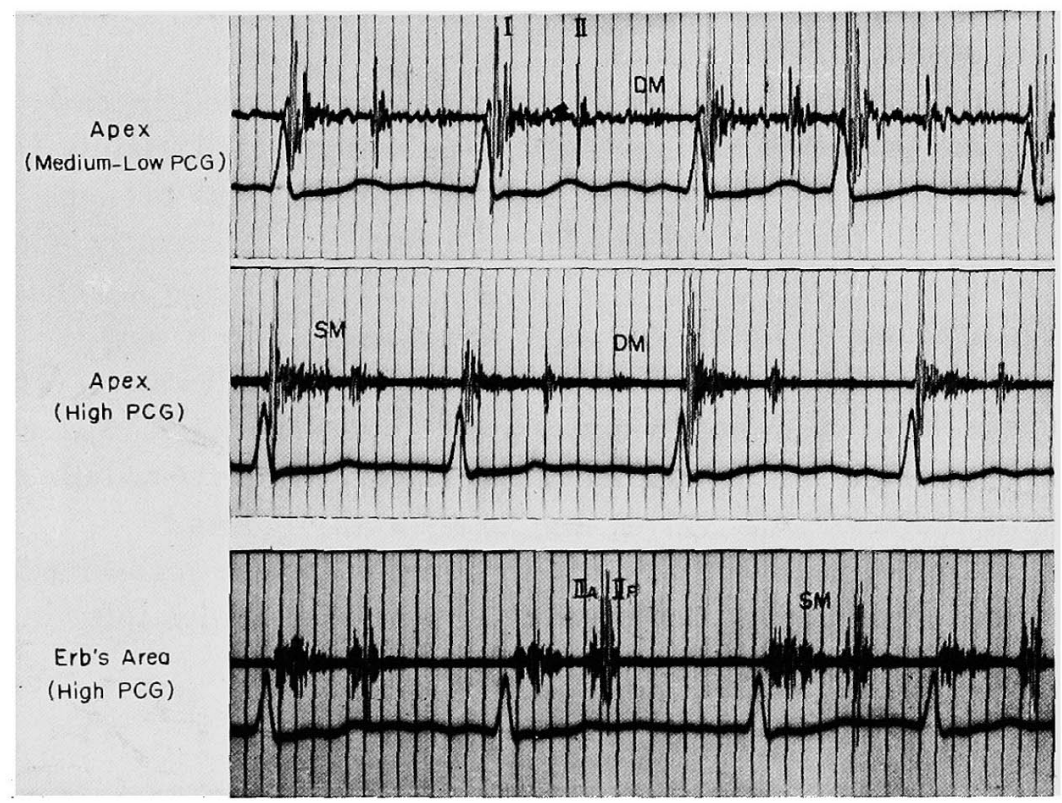

Fig. 15. PCG of a 54-year-old man with hyperthyroidism and atrial fibrillation, showing similar auscultatory findings to mitral stenosis. Basal metabolic rate was $+64 \%$.

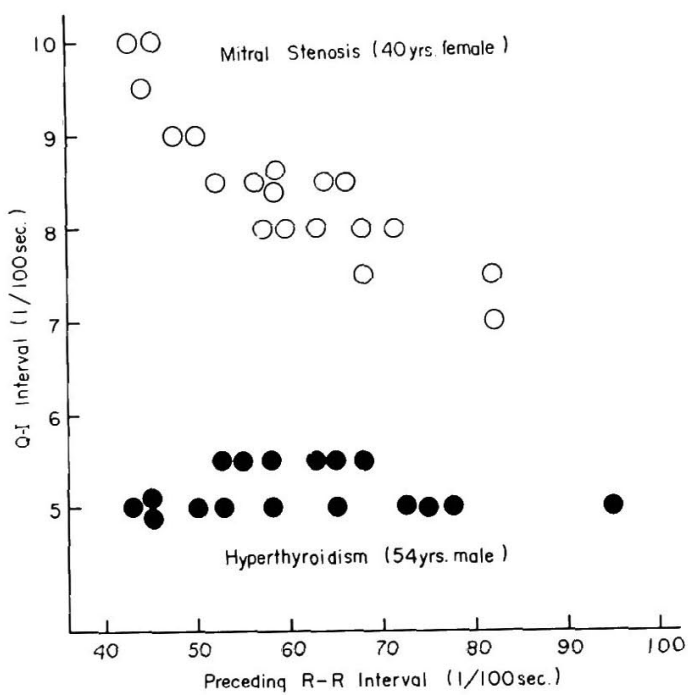

Fig. 16. An example of relationship between $Q-I$ interval and preceding $\mathrm{R}-\mathrm{R}$ interval in hyperthyroidism and mitral stenosis. 
the pulmonic component was louder than the aortic one. The Q-II interval was shorter by $0.05 \mathrm{sec}$. than the Q-T interval.

Case 2. A man, aged 54, was admitted with the complaints of dyspnea and edema on the lower extremities. The chest roentgenogram revealed moderate enlargement of the heart with slight dilatation of the left atrium, and ECG showed atrial fibrillation, incomplete right bundle branch block and ST-T depression in leads II, III, ${ }_{a} V_{F}$ and left precordial leads.

On auscultation, IS was accentuated, and a rough systolic murmur and a rumbling diastolic murmur were heard at the apex. IIS was loud and a systolic murmur was audible at the base. However, in PCG of Fig. 15, there was no opening snap recorded and the Q-I interval was within the normal limits. Furthermore, as seen in Fig. 16, the Q-I interval was not influenced by

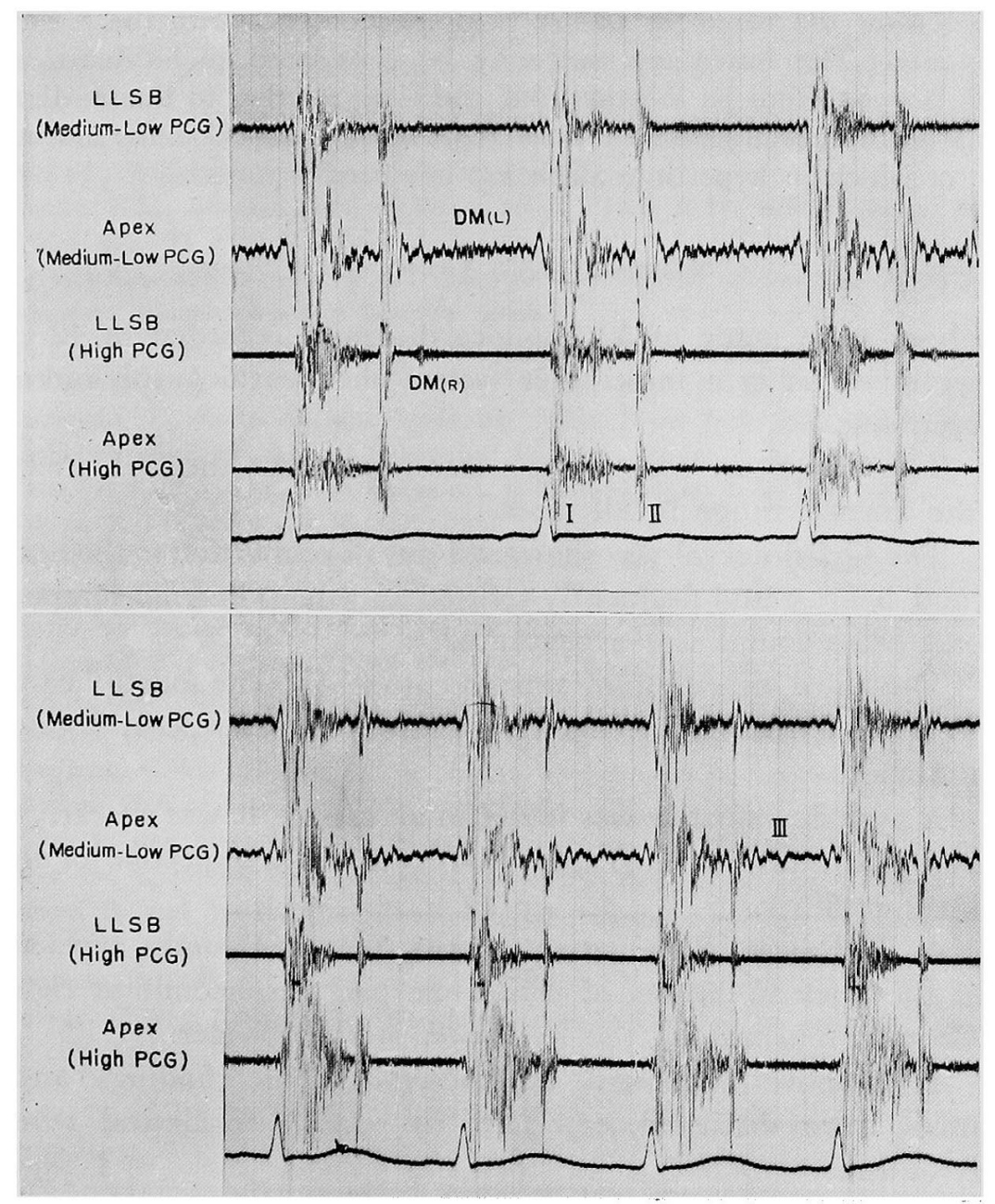

Fig. 17. PCG of a 32-year-old man with two varieties of diastolic murmur. Upper : PCG before treatment (basal metabolic rate $+61 \%$ ). Lower: PCG after treatment for 3 weeks (basal metabolic rate $+44 \%$ ). 
the preceding $\mathrm{R}-\mathrm{R}$ interval, as observed in mitral stenosis. Laboratory examinations disclosed hyperthyroidism with basal metabolic rate of $+64 \%$, I $\mathrm{I}^{131}$ uptake of $87.8 \%$ and protein bound iodine of $1.5 \gamma / 100 \mathrm{ml}$.

The mid-diastolic murmur disappeared and both IS and II S decreased in intensity after treatment for hyperthyroidism.

Case 3. A 32-year-old male had dyspnea, liver swelling and edema on the lower extremities, which had been aggravated 2 or 3 weeks before admission. The chest roentgenogram showed moderate enlargement of the heart, especially of the right heart. The shortening of the mechanical systolic period relative to the prolonged Q-T interval was marked, as seen in PCG of Fig. 17. In addition to a low-pitched mid-diastolic murmur at the apex, a higher-pitched and earlier diastolic murmur was found at the fourth left intercostal space. After administration of antithyroid drug for 3 weeks, the latter murmur disappeared and the former decreased in intensity in concomitance with the reduction of the heart size. The latter murmur may be considered to be due to relative tricuspid stenosis from its location and the time relation to the cardiac cycle. This was an interesting case with the right-sided Carey Coombs murmur, the presence of which in hyperthyroidism has not been reported.

\section{Summary and Conclusion}

Analyses were made of 52 phonocardiograms recorded in 42 cases of hyperthyroidism using a multi-filter system phonocardiograph and a dynamic microphone.

(1) The first heart sound was accentuated, but the Q-I interval fell within the normal range in all cases.

(2) The Q-II interval was shortened out of proportion to the heart rate. The second heart sound frequently splitted and the pulmonic component of the second heart sound was accentuated.

(3) The third heart sound was accentuated. The fourth heart sound had a trend of accentuation, but it was inaudible except in 3 cases with presystolic click.

(4) A systolic murmur was found in all cases. It was an early peaked ejection systolic murmur in graphic configuration irrespective of the area of maximal intensity.

(5) A mid-diastolic murmur of the Carey Coombs type was disclosed at the apex in 7 cases, of which one had a concomitant right-sided Carey Coombs murmur on the left lower sternal border.

(6) The above-mentioned phonocardiographic findings and their significances were discussed, especially in relation to clinical severity of hyperthyroidism.

The mid-diastolic murmur may be considered to be due not only to absolutely increased flow load, but to relatively increased overloading on the damaged heart, which was clinically suggested from the enlarged 
heart and electrocardiographic changes. The differentiation from mitral stenosis was easily made by the following observations; the normal Q-I interval, the reduced mechanical systolic period, the absence of openning snap, the Carey Coombs type of the diastolic murmur and the response of the heart sounds and murmurs to antithyroid drug.

\section{AGKNOWLEDGEMENT}

We are much obliged to Dr. G. Kaito and T. Sakamoto for their advice and encouragement.

\section{REFERENCES}

1. Wood, P.: Diseases of the Heart and Girculation, 2 nd ed., J.B. Lippincott, Philadelphia, 1960.

2. Luisada, A.A.: Heart. A Physiologic and Clinical Study of Cardiovascular Diseases, 2nd ed., Williams and Wilkins, Baltimore, 1954.

3. Renflo, N.L., Leonard, J.J., de Groot, W.J., Paley, H.W., and Coleman, S.L.: Circulation 22: 799, 1960 (Abstract).

4. Levine, S.A. and Harvey, W.P.: Clinical Auscultation of the Heart, 2nd ed., W.B. Saunders, Philadelphia and London, 1959.

5. McKusick, V.A.: Cardiovascular Sound in Health and Disease, Williams and Wilkins, Baltimore, 1958.

6. Sakamoto, T., Kaito, G., and Ueda, H.: Jap. Heart J. 1: 213, 1960.

7. Ueda, H., Uozumi, Z., and Sakamoto, T.: Jap. Heart J. 2: 426, 1961.

8. Ueda, H., Uozumi, Z., and Sakamoto, T.: Tap. Heart J. 3: 207, 1962.

9. Kühns, K.: Gardiologia 23 : 305, 1955.

10. Graettinger, J.S., Muenster, I.J., Selverstone, L.A., and Campbell, J.A.: J. Clin. Invest. 38 : 1316, 1959.

11. Ueda, H., Sakamoto, T., Kaito, G., Watanabe, H., Uozumi, Z., Nakajima, A., and Kobayashi, T.: Nippon-Rinshō 17: 1366, 1959 (in Japanese).

12. Levine, S.A.: Clinical Heart Disease, 5 th ed., W.B. Saunders, Philadelphia and London, 1956.

13. Friedberg, C.K.: Disease of the Heart, 2 nd ed., W.B. Saunders, Philadelphia and London, 1956.

14. Mohr, L. und Staehelin, R.: Handbuch der Inneren Medizin, 4te Aufl,, 9ter Band, Herz und Kreislauf, Springer, Berlin, Göttingen und Heiderberg, 1960.

15. Scherf, D. and Boyd, L.J.: Cardiovascular Diseases, 3 rd ed., Grune and Stratton, New York and London, 1958.

16. Leatham, A.: Brit. Heart J. 17: 574, 1955.

17. Parade, G.W.: Dtsch. med. Wschr. II : 1799, 1935. cited by Mohr, L, and Staebelin, R. (14). 